\title{
One-Stop Management to Initiate Thrombolytic Treatment on the Computed Tomography Table: Adoption and Results
}

\author{
Michal Haršány, a,b Michal Bar, ${ }^{\mathrm{c}}$ David Černík, ${ }^{\mathrm{d}}$ Roman Herzig, ${ }^{\mathrm{e}}$ René Jura, ${ }^{\mathrm{f}}$ Lubomír Jurák, ${ }^{\mathrm{g}}$ \\ Jiří Neumann, ${ }^{\mathrm{h}}$ Daniel Šaňák, ${ }^{\mathrm{i}}$ Svatopluk Ostrý, ${ }^{\mathrm{j}}$ Petr Ševčík, ${ }^{\mathrm{k}}$ Ondřej Škoda, ${ }^{\mathrm{l}}$ David Školoudík, ${ }^{\mathrm{m}}$ \\ Daniel Václavík, ${ }^{\mathrm{n}}$ Aleš Tomek, ${ }^{\circ}$ Robert Mikulík, ${ }^{\mathrm{a}, \mathrm{b}}$ on Behalf of the Czech Stroke Unit Network \\ ${ }^{a}$ Department of Neurology, St. Anne's University Hospital in Brno, Faculty of Medicine Masaryk University, Brno, Czech Republic \\ 'International Clinical Research Centre, St. Anne's University Hospital in Brno, Brno, Czech Republic \\ 'Department of Neurology, University Hospital Ostrava, Faculty of Medicine at University Ostrava, Ostrava-Poruba, Czech Republic \\ ${ }^{d}$ Comprehensive Stroke Center, Department of Neurology, Masaryk Hospital Ústí nad Labem, KZ a.s., Ústi nad Labem, Czech Republic \\ 'Department of Neurology, Charles University Faculty of Medicine and University Hospital, Hradec Kralove, Czech Republic \\ fDepartment of Neurology, University Hospital Brno, Faculty of Medicine Masaryk University, Brno, Czech Republic \\ ${ }^{9}$ Neurocentre, Regional Hospital Liberec, Liberec, Czech Republic \\ hDepartment of Neurology and Stroke Centre, Krajská zdravotni-Hospital Chomutov, Chomutov, Czech Republic \\ 'Comprehensive Stroke Center in Department of Neurology, Palacky Medical School and University Hospital, Olomouc, Czech Republic \\ 'Comprehensive Stroke Centre, Hospital České Budějovice a.s., České Budějovice, Czech Republic \\ kDepartment of Neurology, University Hospital Pilsen, Faculty of Medicine in Pilsen, Charles University, Plzen, Czech Republic \\ 'Department of Neurology, Vinohrady University Hospital, 3rd Medical School of Charles University, Prague, Czech Republic \\ ${ }^{m}$ Center for Science and Research, Faculty of Health Sciences, Palacký University Olomouc, Olomouc, Czech Republic \\ nDepartment of Neurology and Agel Research and Training Institute, Ostrava Vitkovice Hospital, Ostrava, Czech Republic \\ 'Department of Neurology, Motol University Hospital, 2nd Medical School of Charles University, Prague, Czech Republic
}

\section{Dear Sir:}

Data from clinical practice show that median door-to-needle time (DNT) for intravenous thrombolysis (IVT) remains around 60 minutes. ${ }^{1}$ However, many reports documented that hospital logistics could be substantially streamlined and DNT shortened below 30 minutes. ${ }^{2}$ Given Czech stroke care reform, many stroke centers have recently been changing their logistical pathways with the aim to initiate IVT quicker than before. ${ }^{3}$ Several stroke centers introduced one-stop management, which means that all suspected acute ischemic stroke (AIS) patients are admitted directly to the computed tomography (CT) room and IVT is initiated on the CT table. As a consequence, our national data show that some hospitals were able to reach an extremely short DNT, while others were not. The aim of this nationwide study was to understand how logistical pathways are being used, and how they impact quality of patient care as measured by DNT.
This is a nationwide prospective study combining hospital-level data from a questionnaire and patient-level data from the Safe Implementation of Treatments in Stroke (SITS) registry from January 2017 to March 2018 to define differences in logistical pathways for IVT between stroke centers, and to analyze the impact of such differences on DNT. A description of the questionnaire, collection of data on DNT from the SITS registry and statistical analysis is shown in the Supplementary methods. ${ }^{2,47}$

All 45 stroke centers in the Czech Republic responded. Of these, six centers reported one reorganization and two centers reported two reorganizations in their acute stroke care. During the study period, four centers introduced one-stop management and following differences in median DNT before versus after introduction of one-stop management were reported: 30 minutes vs. 17.5 minutes, 30 minutes vs. 28.5 minutes, 30 minutes vs. 20 minutes, and 45 minutes vs. 28 minutes. The change of proportion of patients with DNT $\leq 20$ minutes in 
these centers is shown in the Supplementary Table 1. Thus, 55 center-datasets were included in the analysis. Altogether, 5,889 patients were treated with IVT in the study period and had available DNT in the SITS registry. Median DNT $\leq 20 \mathrm{~min}-$ utes was achieved in $12(22 \%)$ centers. Overall, $18(33 \%)$ centers reported direct patient admission to CT room, 26 (47\%) admission to emergency department, and 11 (20\%) to out-patient office. Notably, median DNT was 20 minutes (interquartile range [IQR], 18 to 26$)$ in centers without any transfers, 28 minutes (IQR, 22 to 30 ) with one transfer and 37 minutes (IQR, 30 to 43) with two transfers before initiation of IVT. Data on stroke logistics stratified based on the place of admission are shown in Supplementary Table 2.

Our study investigated current practice in logistics of IVT in a nationwide sample in the Czech Republic, a country with a large number of thrombolytic treatments and a median national DNT of only 28 minutes in 2017 to 2018. During this period, all stroke centers already met the target of 60 minutes recommended by the most recent guidelines ${ }^{8}$ and this happened regardless of differences in stroke pathways. Within the short national DNT, there were still differences in the DNT between stroke centers, with approximately one-fifth of hospitals achieving ultrashort median DNT $\leq 20$ minutes. Logistical pathways in stroke centers have been substantially restructured in many stroke centers allowing direct transport of AIS patients into CT room. Such change increased proportion of patients with DNT $\leq 20$ minutes from $28 \%$ to $54 \%$. We found that in one-third of stroke centers AIS patients were still transferred two times within a hospital before initiation of IVT which resulted in nearly doubled DNT compared to hospitals where patients were admitted directly to the CT room and treated on the CT table (37 minutes vs. 20 minutes).

The limitations of our study are inherent to a survey based on a questionnaire. We cannot exclude that there might have been some inaccuracies in reported data. However, all stroke centers collect data on all patients treated with IVT and these data are reported monthly to the Czech Stroke Society and annually to the Ministry of Health. It is also unlikely that hospitals would have provided an incorrect description of their usual logistical pathway, although this pathway does not necessarily apply to all cases. Furthermore, safety and efficacy of ultrafast delivery of recombinant tissue plasminogen activator needs to be documented and is a subject of our ongoing analysis. Applicability of direct transports to $\mathrm{CT}$ room is possible only in those countries, which have well functioning emergency medical service (EMS) and a network of stroke centers with adequate capacities and training. In the Czech Republic, EMS always transfers AIS patients to neurologists and there are no legal barriers for direct transfers to CT rooms. As at least one previous study demonstrated national median DNT of 25 minutes, ${ }^{2}$ our results are independent from specifics of one country's health care system and should be thus generalizable also to other countries. In our study we demonstrated benefits of direct transport to CT rooms but it is also possible to transfer patients directly to an angio-suite. Such logistics was not reported in our country. Although previous reports documented shortened door-to-groin time for mechanical thrombectomy, generalizability could be even more challenging especially due to technical requirement for angiograms, and availability of interventional team in short time manner. $^{9}$

In conclusion, one-third of hospitals nationally adopted onestop management of IVT on the CT table and this approach provided thrombolytic treatment faster than any other mode of logistics.

\section{Supplementary materials}

Supplementary materials related to this article can be found online at https://doi.org/10.5853/jos.2021.00878.

\section{References}

1. Xian $Y, X u H$, Lytle B, Blevins J, Peterson ED, Hernandez AF, et al. Use of strategies to improve door-to-needle times with tissue-type plasminogen activator in acute ischemic stroke in clinical practice: findings from target: stroke. Circ Cardiovasc Qual Outcomes 2017;10:e003227.

2. Kuhrij LS, Wouters MW, van den Berg-Vos RM, de Leeuw FE, Nederkoorn PJ. The Dutch acute stroke audit: benchmarking acute stroke care in the Netherlands. Eur Stroke J 2018;3: 361-368.

3. Bryndová L, Bar M, Herzig R, Mikulik R, Neumann J, Šaňák D, et al. Concentrating stroke care provision in the Czech Republic: the establishment of stroke centres in 2011 has led to improved outcomes. Health Policy 2021;125:520-525.

4. Meretoja A, Strbian D, Mustanoja S, Tatlisumak T, Lindsberg PJ, Kaste M. Reducing in-hospital delay to 20 minutes in stroke thrombolysis. Neurology 2012;79:306-313.

5. Meretoja A, Weir L, Ugalde $M$, Yassi N, Yan B, Hand $P$, et al. Helsinki model cut stroke thrombolysis delays to 25 minutes in Melbourne in only 4 months. Neurology 2013;81:1071-1076.

6. Topakian R, Hörmanseder B, Einsiedler S, Straka U, Oel D, Metschitzer $B$, et al. Ultra-short door-to-needle times of 10 minutes or less in stroke thrombolysis: experience from 63 cases (S8.002). Neurology 2017;88(16 Suppl):S8.002.

7. Ebinger M, Siegerink B, Kunz A, Wendt M, Weber JE, Schwa- 
bauer $E_{1}$ et al. Association between dispatch of mobile stroke units and functional outcomes among patients with acute ischemic stroke in Berlin. JAMA 2021;325:454-466.

8. Powers WJ, Rabinstein AA, Ackerson T, Adeoye OM, Bambakidis NC, Becker K, et al. 2018 Guidelines for the early management of patients with acute ischemic stroke: a guideline for healthcare professionals from the American Heart Association/American Stroke Association. Stroke 2018; 49:e46-e110.

9. Psychogios MN, Maier IL, Tsogkas I, Hesse AC, Brehm A, Behme $D$, et al. One-stop management of 230 consecutive acute stroke patients: report of procedural times and clinical outcome. J Clin Med 2019;8:2185.
Correspondence: Michal Haršány

Department of Neurology, St. Anne's University Hospital in Brno, Faculty of Medicine Masaryk University, Pekarska 53, Brno 65691, Czech Republic Tel: +420-720466696

Fax: +420-543182624

E-mail: michal.harsany@fnusa.cz

https://orcid.org/0000-0003-4319-133X

Received: March 9, 2021

Revised: June 5, 2021

Accepted: July 21, 2021

Michal Haršány and Robert Mikulik have been supported by the project no. LQ1605 from the National Program of Sustainability II (MEYS CR); Robert Mikulik has been supported by the COST (European Cooperation in Science and Technology) Association, project No. CA18118, IRENE COST ActionImplementation Research Network in Stroke Care Quality and by the IRIS-TEPUS Project No. LTC20051 from the INTER-EXCELLENCE INTER-COST program of the Ministry of Education, Youth and Sports of the Czech Republic; Roman Herzig has been supported by the projects DRO-UHHK00179906 and PROGRES-040.

We would like to kindly thank Steven Simsic for proofreading the manuscript. 


\section{Supplementary methods}

\section{Questionnaire}

The questionnaire inquired about in-hospital logistical processes preceding application of intravenous thrombolysis (IVT) during the study period. Prior to the study, the questionnaire was approved by the Czech Stroke Society (formally Cerebrovascular Division of the Czech Neurologic Society). The questionnaire assessed prenotification, place of admission where patients are brought by an ambulance within hospital (computed tomography [CT] room, emergency department [ED] or out-patient office), number of transfers, drawing blood, and other examinations before initiation of thrombolytic treatment, imaging protocol before initiation of IVT (noncontrast computed tomography [NCCT] vs. NCCT and computed tomography angiography [CTA] vs. NCCT, CTA and computed tomography perfusion [CTP], respectively), and place where IVT is initiated (ED, on the $\mathrm{CT}$ table, etc.).

A transfer was defined as transport from one place in the hospital to another place even if places were close to each other (e.g., admission to ED and transport to CT scanner, even if CT scanner was within ED, was counted as one transfer). Blood draw in our study meant that a stroke team drew a blood sample without waiting for the results (if not indicated). Other examinations preceding initiation of IVT were defined as physical examination (except for neurological examination), performing electrocardiogram or urinary catheter insertion. A reorganization was defined as a change in steps preceding initiation of IVT assessed by the questionnaire.

All stroke centers in the Czech Republic were asked to participate by email. If a stroke center did not respond within 3 months, the email was re-sent. After an additional 3 months there was no response from four centers, and seven centers provided incomplete data. From these 11 centers, complete data were obtained after repeated requests and phone calls.

\section{Safe Implementation of Treatments in Stroke registry}

Data on door-to-needle time (DNT) for each patient were obtained from the Safe Implementation of Treatments in Stroke (SITS) registry. If a center reported a reorganization of acute stroke care, DNT was calculated separately for each time period. DNT was defined as the time from hospital arrival to the initiation of IVT. In the Czech Republic, DNT has been prospectively collected for each patient in different registries (SITS and Registry of Stroke Care Quality [RES-0]) and also annually reported to the Ministry of Health as summary statistics.

The SITS-Monitoring Study was approved by the Ethics Committee of the Karolinska Institute in Stockholm. Analysis of the data from the SITS registry was approved by St. Anne's University Hospital Ethics Committee. Informed consent was not required because data were collected as part of an audit of clinical care.

\section{Statistical analysis}

The outcome for the analysis was DNT $\leq 20$ minutes, this cutoff was chosen based on previously published data. ${ }^{2,4-7}$ Relationships between DNT $\leq 20$ minutes and all variables were analyzed using the Kruskal-Wallis test for numerical parameters and the chi-square test for nominal and categorical parameters. Centers were stratified according to place of admission and number of transfers. All statistical analyses were performed using IBM SPSS Statistics software version 23 (IBM Co., Armonk, NY, USA). 
Supplementary Table 1. The proportion of patients with DNT $\leq 20$ minutes in the centers before and after the introduction of one-stop management

\begin{tabular}{lccc}
\hline & All patients with DNT $\leq 20$ minutes & DNT $\leq 20$ minutes minutes before reorganization & DNT $\leq 20$ minutes after reorganization \\
\hline Center 1, N & $186(44)$ & $80(34)$ & $106(57)$ \\
Center 2, N & $25(17)$ & $19(14)$ & $6(38)$ \\
Center 3, N & $31(34)$ & $26(32)$ & $5(56)$ \\
Center 4, N & $0(0)$ & $0(0)$ & $0(0)$ \\
\hline
\end{tabular}

Values are presented as number (\%).

DNT, door-to-needle time.

Supplementary Table 2. Stroke logistics in centers according to place of admission

\begin{tabular}{lccc}
\hline Variable & CT room $(n=18)$ & Emergency department $(n=26)$ & Out-patient office (n=11) \\
\hline DNT (min) & $22(18-26)$ & $30(25-35)$ & $30(24-39)$ \\
DNT $\leq 20$ minutes & $9(50)$ & $2(8)$ & $1(9)$ \\
Prenotification & $18(100)$ & $22(85)$ & $10(91)$ \\
No. of transfers & & & $0(0)$ \\
$\quad$ None & $16(89)$ & $0(0)$ & $5(45)$ \\
1 & $2(11)$ & $14(54)$ & $6(55)$ \\
2 & $0(0)$ & $12(46)$ & $8(73)$ \\
Blood draw & $14(78)$ & $24(92)$ & $2(18)$ \\
Other examinations & $3(17)$ & $15(58)$ & $2(18)$ \\
Imaging protocol & & & $7(64)$ \\
NCCT & $4(22)$ & $7(27)$ & $2(18)$ \\
NCCT+CTA & $12(67)$ & $17(65)$ & $5(45)$ \\
NCCT+CTA+CTP & $2(11)$ & $14(54)$ & \\
IVT on CT table & $16(89)$ & & \\
\hline
\end{tabular}

Values are presented as median (interquartile range) or number (\%).

CT, computed tomography; DNT, door-to-needle time; NCCT, noncontrast computed tomography; CTA, computed tomography angiography; CTP, computed tomography perfusion; IVT, intravenous thrombolysis. 